\title{
LA ESCRITURA NERUDIANA DESDE LA POÉTICA DE MIJAIL BAJTIN
}

\author{
Manuel Jofré \\ Universidad de Chile \\ jofremanuel@gmail.com
}

RESUMEN / ABSTRACT

Se establece aquí un paralelo entre la vida de Mijail Bajtin y Pablo Neruda. Se toman luego las categorías bajtinianas de monologismo y dialogismo como fuerzas que polarizan la poesía de Neruda. En seguida, se estudian los cronotopos que arqui-texturan esta poesía y finalmente, se aplican las cuatro categorías de la historia de la novela de Bajtin a la compleja sucesión de la escritura del poeta.

Palabras clave: Neruda, Bajtin, monologismo, dialogismo, cronotopo, realismo.

This article begins with a parallel between Mijail Bajtin and Pablo Neruda's lives and proceeds with a discussion of bajtinian categories of monologism and dialogism, which are viewed as forces that tension Neruda's poetry. Finally, Bajtin's four categories of the history of the novel are applied to the complex succession of the poet's writing.

KEY WORDS: Bajtin, monologism, dialogism, chronotope, realism.

No se ha intentado describir el universo escritural nerudiano desde el punto de vista de la poética de Mijail Bajtin, el más importante teórico de la literatura ruso del siglo XX. Pablo Neruda vivió entre 1904 y 1973, mientras que Mijail Mijailovich Bajtin lo hizo entre 1895 y 1975. Vidas paralelas en el tiempo pero en espacios diferentes: Neruda mayormente en América Latina y Bajtin casi exclusivamente en Rusia (hay dudas sobre su supuesto viaje a Italia en 1928). Ambos escritores, pero de dimensiones muy diferentes. Neruda publicó 
casi 60 libros y Bajtin solo 5. El primero, altamente conocido, a lo largo de su vida; el segundo, un desconocido, toda su vida.

\section{UN PARALELO (IM)POSIBLE}

En un ensayo breve es difícil poder capturar toda la magnitud de una escritura. Pero igualmente interesa comparar y contrastar su praxis discursiva. Bajtin fue un prosista ideólogo, en sus propios términos. Neruda, por su parte, más bien un poeta de sensaciones. Sin embargo, ambos orientaron su escritura hacia lo material. Bajtin reformuló varias veces la historia de la literatura occidental, mientras que Neruda la proyectó en direcciones no pensadas previamente.

Neruda fue un escritor práctico, pero lírico (aunque narrativo en muchas ocasiones), no interesado en la teoría de la literatura. Bajtin, por su parte, se estableció como un teórico de la prosa más que un analista de la poesía. Ambos se situaron en géneros diferentes, y tal vez opuestos. Sin embargo, ambos, desde dominios lingüísticos diferentes, y por caminos muy distintos, apuntaron a una totalización discursiva. La asimilación que ambos hacen de la literatura occidental previamente acumulada es formidable. Desde el punto de vista político, por otro lado, sus visiones son contrapuestas. Neruda recibió gran atención del oficialismo soviético, el cual aplaudió sin críticas, mientras que Bajtin fue sometido a un injusto proceso político (autoritarismo de ultraizquierda, en 1928), que pretendió la anulación de su escritura, felizmente sin obtenerlo. Por otra parte, 20 años antes, el propio Neruda sufrió los avatares del autoritarismo de derecha, a fines de los años 50.

El objetivo de este trabajo inicial no es establecer el paralelo imposible pero realizable entre dos grandes figuras escriturales del siglo XX. Neruda abrazó tardíamente (a los 41 años) el marxismo ortodoxo de orientación rusa predominante, mientras que Bajtin jamás se encontró con esta matriz autoritarista, formulando, en cambio, un sistema teórico alternativo mucho más creativo y alternativo al marxismo absolutista estaliniano de la Unión Soviética.

Con su poesía, Neruda ciertamente orienta la palabra poética en relación con los oprimidos por el sistema capitalista dominante y, por cierto, sin duda alguna, lo mismo puede decirse de la escritura bajtiniana, la cual tiene su base en la experiencia personal sufriente y en la experiencia alegre colectiva. Ambos buscaron, efectivamente, el mejoramiento de la humanidad y de su historia de tragedias y violencias, por distintas articulaciones, pero utilizando el mismo instrumento, la palabra humana. 
Lo más posible es que Neruda nunca se haya enterado de la existencia de Mijail Bajtin, aunque Bajtin sí supo de la poesía de Neruda publicada en la Unión Soviética. Ambos eran intelectuales anti-establishment, en el pleno sentido de la expresión, fundando mundos para el futuro de los otros mediante la palabra y, por cierto, sus escrituras aún no han sido asimiladas por la historia humana.

Los objetivos de la escritura de ambos escritores, si acaso formulables, eran muy disímiles. Bajtin escribió lúcidos análisis de la obra de Rabelais y de Dostoievski, pero no se propuso lo que sin embargo realizó, que es reescribir varias veces la historia de la literatura de Occidente y, en particular, la historia de la novela como género no canonizado. Neruda transformó, en cambio, todo lo que estaba al alcance de su palabra, desde la naturaleza a lo social, desde lo personal a lo colectivo, desde lo histórico hasta lo mítico, en un proyecto poético amplísimo y cambiante, que poetizaba todo lo que tocaba.

Neruda fue un escritor expansivo, que pudo publicar desde el comienzo todo lo que escribía, con un amplio margen de libertad expresiva. Bajtin, en cambio, desde sus primeros escritos, se vio obligado a cuidar su escritura, escondiéndola, incluso evitando su diseminación, y, por tanto, reconcentrándola, compactándola, y escribiendo entre líneas, para poder preservarla.

Con este horizonte inicial para ambos escritores, es que, con riesgo y con delicadeza, se intenta aquí no mirar a Bajtin desde Neruda, sino que contemplar a Neruda desde Bajtin, acto dispar pero posible ${ }^{1}$.

\section{MONOLOGISMO Y DIALOGISMO}

La poética nerudiana necesita ser visualizada desde las nuevas perspectivas teóricas de avanzada. Las ideas de Bajtin, una parte de ellas, pueden ser propicias para esta tarea. Desde el punto de vista de la discursividad, una primera interrogante es acerca del carácter mayoritariamente monológico de la escritura nerudiana. Esta voz única posee una modalidad constante, uniforme, homogénea. Lo monológico de la escritura nerudiana se basa en lo unitario de su obra, en el autocentramiento del poeta, en la uniformidad

1 Nuestro planteamiento sobre la obra nerudiana ha quedado registrado en Jofré, Pablo Neruda: de los mitos y el ser americano. 
de la tonalidad ${ }^{2}$. El tono, según Bajtin, es lo más personal y propio de la comunicación humana.

Aunque el hablante nerudiano intenta dialogar con su amada en su poesía juvenil, ella, como se ha notado, no está presente ni corresponde a dicha afectividad. La amada no responde y por eso, monológicamente, los Veinte poemas de amor y una canción desesperada (1924) son de desamor, cuya tonalidad solitaria los permea y estructura. Frente a esta tristeza y melancolía imperante emerge, sin embargo, "La canción de la fiesta", con lo dionisíaco, corporal y carnavalesco.

La salida del lenguaje monológico acontece mediante la otredad y el dialogismo. Si a Bajtin le pareció que la poesía de Neruda era parte del realismo grotesco, lo afirmó así porque visualizaba que su obra (lo que Bajtin conocía, y no está claro si el juicio fue escrito en 1946 o en 1965) se escapaba de la línea discursiva occidental, descrita como predominantemente monológica (es decir, idealizada, estilizada, uniforme, monoestilística). Importa mucho en este punto destacar cómo la obra de Neruda comienza tempranamente a incluir las preguntas, interrogantes, cuestionamientos, como macrogénero discursivo, en multiplicidad de poemas, incluso antes de la modelación del aporte cronotópico (ciclo de la materia y ciclo del tiempo), dirigidos tanto a sí mismo como al lector empírico. Esto abarca, por cierto, tanto lo epistemológico (el saber sobre el mundo) como lo ontológico (el ser de la realidad).

La poesía de Neruda emerge de la tensión entre el monologismo y el dialogismo, que luchan por manifestarse en la escritura del poeta. Eso lo posibilita el carácter crítico de su poesía, su radical postura disconforme, la postulación de la poesía como discurso alternativo. La postura crítica en el universo verbal nerudiano consiste en un rechazo al tiempo cósmico destructor de la materia (visión que impera hasta la segunda residencia) y en un segundo rechazo a la degradación en la estructura social jerárquica y burocrática como rasgo definitorio de lo chileno. Neruda comienza construyendo una versión alternativa a la verdad oficial, desde su prehistoria poética (1915-1920).

Canto general (1950) puede ser considerado un primer momento de salida del monologismo que caracterizó las etapas previas a 1936. Hasta ese momento, Neruda había desarrollado una filosofía del tiempo material cósmico y estaba a punto de generar una filosofía del tiempo histórico y social.

2 Respecto de lo monológico versus lo dialógico, véase Bakhtin, Problems of Dostoevsky's Poetics. 
Canto general intentó quebrar el monologismo mediante tres dispositivos posibles de diferenciar. Primero, porque se está escribiendo una historia alternativa de América Latina, centrada en la búsqueda de libertad y democracia, y enfocada en los actores que siempre habían sido marginales (indios, patriotas, libertadores, igualitaristas). Segundo, el poeta considera como fundamental la voz del otro (el indígena, el insurgente y el trabajador), y decide, consecuentemente, por la teoría poética de ser el representante de los otros, incorporando sus palabras (las palabras ajenas) en los poemas propios. Es así como la voz del pueblo llega a resonar por primera vez en Canto general. Esa preocupación por lo social y natural humano se incrementará en las Odas elementales (1954). Tercero, un mecanismo de asunción del otro en Canto general (el más desarrollado) es reproducir en una cita, insertándola, lo dicho por obreros, mineros, campesinos, trabajadoras. Con ello, Neruda se abre a la historia del otro, al discurso del marginado y a la problemática del excluido.

Neruda luchó para encontrar, mediante su escritura, una forma poética legítima de incorporar la otredad y lo social. Esto se hace más fuerte a partir de Estravagario (1958). Para ello, prueba lo jocoso, lo semicómico, lo paradójico, la unión de los contrarios, la autoironía. Ya en las Odas se ríe de los viejos poetas y del discurso nostálgico y narcisista de los poetas tradicionales.

No se ha estudiado suficientemente en Neruda el procedimiento del desdoblamiento (como rememoración en Memorial de Isla Negra, al centrarse "en el otro que fui", "los muchos que soy") como fórmula para dialogizar la escritura poética. Este camino funciona de otro modo en la fábula mitológica de La espada encendida (1970), que contribuye a la idea de que el yo nerudiano (monológico) disimula o enmascara a un otro, lo que dialogiza la situación. Así, el poeta es un yo, hecho de múltiples voces, más su proyección y recuperación de la otredad.

Finalmente, el poemario más dialógico de todas las obras de Neruda es El libro de las preguntas (1974), donde la interrogante monológica (como género discursivo que presupone y consulta al otro) lucha por generar una respuesta de parte del lector. Por fin, el macrogénero conativo, interpelativo, de las preguntas alcanza su máxima expresión (las interrogantes decisivas ya estaban en "Galope muerto" y "Explico algunas cosas"). Hay aquí una concreción dialógica de la estética de la recepción, que incorpora al narratario de los poemas-preguntas y al lector empírico mismo. El poema rompe sus propios límites y es solo una parte del gran diálogo en desarrollo. Eso permite un renacer de lo lírico como algo absurdo y antipoético, en El libro de las preguntas. 
Todos los libros de Neruda contribuyen a la búsqueda constante, por parte del héroe interiormente diseñado, de un nivel humano y material más pleno, más íntegro. La formación del héroe y sus etapas, personal y colectivo, que destaca en la poesía nerudiana, debe ser examinada con respecto a la gran narrativa construida por la especie humana. Neruda y su poesía contribuyen a desarrollar y profundizar este gran megarrelato que es la historia de la literatura, de la conciencia y de las personas.

Neruda es pues un refundador de lo nuestro humano. Chile y Neruda están indisolublemente unidos ante todas las otras culturas y naciones. Latinoamérica es la otra dimensión con la cual dialoga. Por eso, cuando nuestras naciones están cumpliendo más de 200 años de vida independiente, la obra nerudiana emerge como un gran faro guía. En la celebración de los Bicentenarios, y en la caída de las estructuras anquilosadas y añejas del siglo XX, Neruda es una fuerza cósmica y humana creativa, cuyo aporte pertenece a todos. Nosotros somos los interlocutores de Neruda, con los cuales dialogizó su palabra poética y humana, con la risa e ironía de su penúltimo gran poema, "Autorretrato". Una primera cartografía de lo monológico versus lo dialógico en la poesía de Neruda, oposición por primera vez consignada por Bajtin, que nos permite los resultados entregados.

\section{CONCLUSIONES INICIALES CON CRONOTOPO}

El propósito central en este breve trabajo ha sido abordar la obra poética de Neruda en su conjunto. Esto solo es posible luego de una amplia variedad de estudios realizados con respecto a los múltiples poemarios nerudianos. La óptica general de encuadre ha sido la "poética histórica" de Bajtin, en un sentido amplio. El sistema bajtiniano se manifiesta a través de una serie de formulaciones diferentes, contenidas en sus distintos libros (y sus re-escrituras) y sus ensayos.

La noción de cronotopo, acuñada por Bajtin hacia 1937, es de extrema utilidad para los estudios nerudianos. El cronotopo es una noción bifocal y bivocálica, que cruza y articula dos series, la del tiempo y la del espacio; con ello, Bajtin se aleja de la problemática del héroe (el autor y personaje) y de la historia narrada (el discurso y la acción) ${ }^{3}$. La literatura, y la historia de

3 Véase la mejor traducción al español de "Formas del tiempo y el cronotopo en la novela", en Bajtin, Problemas literarios y estéticos. 
la novela, que ha tomado siglos, es la historia del tránsito de los cronotopos más simples hasta los cronotopos más complejos, en su articulación del tiempo-espacio humano. En inglés han comenzado a usarse nociones como 'chronotypes' y 'spime' (space and time).

La tesis central de Bajtin es que el tiempo y el espacio estuvieron alguna vez articuladamente unidos (como los géneros altos y bajos o como los géneros serio-cómicos) y posteriormente, la historia discursiva de la conciencia humana, con su proceso de monologizacion (racionalización idealista y división reificante del trabajo), llevó a una escisión entre ambas nociones.

El cronotopo es esencial porque permite la creación del género, y evidencia el tránsito desde el campo a la ciudad, en su desarrollo, para luego profundizar en los diferentes ámbitos sociales fragmentados. Para la literatura moderna, el cronotopo significa, primero, la búsqueda de la integración de los diferentes tiempos creados por el ser humano, y, segundo, la integración de los diferentes espacios que constituyen la experiencia humana.

Es posible examinar los casi 60 libros de Neruda desde la óptica constructiva de la sucesión de cronotopos. Primero, como tesis central de esta sección, se plantea que el cronotopo nerudiano se estructura a partir de la reflexión poética acerca de la materia y del tiempo, la cual se encuentra fundamentada en la experiencia de los bosques del sur de Chile. El espacio se manifiesta desde el inicio como materia, como sustancia, como cosa, en Neruda. Segundo, las diversas caras, tanto de la materia como del tiempo, aparecen parciales o desintegradas al inicio de la escritura nerudiana. Luego, gracias a la exploración y modulación poética de la sucesión de obras, estas diversas facetas o aspectos vienen a integrarse hacia la madurez, en la parte final de la tercera etapa de la producción nerudiana (1958-1973), justamente en el denominado Neruda postmoderno. Tercero, se evidenciará que en la escritura nerudiana tanto las presencias como ausencias de cronotopos son decisivas. Solo se utilizarán aquí los cronotopos estrictamente señalados y estudiados por Bajtin.

En su etapa juvenil pre-metropolitana o campesina (1915-1919), hay una primera coexistencia de diferentes cronotopos. El cronotopo folclórico presenta la visión de una comunidad local pueblerina enmarcada por la naturaleza y lo rural. Es mayormente masculina y heliocéntrica en unión con una tierra madre generadora. Se concreta así el cronotopo de la pequeña ciudad de provincia con un cronotopo sociocostumbrista, ambos de índole social donde se exploran los espacios de interacción humana. Con ello se conforma un primer retrato social del contexto comunitario. 
Es importante aquí, en esta prehistoria nerudiana, antes de la publicación del primer libro, el cronotopo idílico, en su versión laboral-agrícola, porque ya marca la arquitectónica de una naturaleza armónica. La sociedad es disonante e inarticulada, pero la naturaleza es uniforme y organizada. Finalmente, es decisiva la ausencia del cronotopo del encuentro, es decir, el establecimiento dialogal de una relación amorosa e integral. Esto marca la situación existencial de un héroe aislado, donde lo sensorial es decisivo sin poner énfasis, sin embargo, en el cronotopo biográfico.

En el período 1920-1925, ya en Santiago, en Veinte poemas de amor y una canción desesperada (1924), se enfatiza el cronotopo sentimental, donde el héroe, sin embargo, no logra llevar a buen fin la aventura amorosa. Las pruebas que constituyen este cronotopo de la aventura son los obstáculos y dificultades que impone la sociedad para su realización y, por tanto, se genera una crítica a ella que es, en consecuencia, una manifestación del cronotopo sociocostumbrista, como se expresa en Crepusculario (1923). La sociedad casi es vista como esclavista, degradada, inmesiricorde.

El período en Oriente (1926-1931) es la intensificación del cronotopo de la aventura y del cronotopo sociocostumbrista que ahora abarca las dos caras y culturas planetarias. Residencias testimonia las aventuras y las pruebas en el itinerario óptico y epistemológico frente a la realidad. El héroe individual construye un modelo de lo real, pero no tiene total conciencia de ello. La experiencia de la soledad es sufriente (y corresponde, exacta y paradójicamente, a la experiencia del destierro de Bajtin al norte de Moscú).

Aventura y pruebas se mantienen como los dos cronotopos regidores de la experiencia discursiva entre el regreso de Oriente (1932) y la composición gradual de Canto general (1938 hasta 1949, en sentido amplio, o 1948-1949, en uno más acotado). Es el cronotopo del sociocostumbrismo de aventura. Cabe notar que el cronotopo sociocostumbrista es el que permite la inclusión del contexto extraliterario o sociocultural, el cual está en la base de la denominada poesía política de Pablo Neruda. Casi paralelo a la escritura de Canto general, se manifiesta el cronotopo del encuentro, con los otros, en lo social-colectivo (desde la guerra civil española, en 1936), y que alcanza su culminación, apropiadamente, en "Alturas de Macchu Picchu". Luego, retorna también el cronotopo idílico de la organización armónica entre el ser humano y la naturaleza, ahora como caracterizador del momento originario de los pueblos americanos de los cuales descendemos. Es la cosmogonía de "La lámpara en la tierra", en el primer canto. 
Con un héroe colectivo del cual se es parte (el pueblo), emerge el cronotopo de la novela histórica en Canto general, donde se narra el antagónico drama de la liberación versus el dominio. Mediante la historia (desde 1400 hasta 1949) y la geografía (de norte a sur), se logra la máxima inmersión del héroe en el cronotopo sociocostumbrista y crítico, explorador del pasado de la sociedad latinoamericana y chilena y diagnosticador del presente. Es también la realización del cronotopo de la formación, de la novela de las generaciones (precolombino, colonial, moderno y contemporáneo), con sus héroes indígenas, los patriotas liberadores, los constructores de las repúblicas y los luchadores en el presente (1949).

Hay que destacar que entre 1950 y 1958 hay dos líneas cronotópicas diversificadas, lo que no había acontecido anteriormente. Primero, la del cronotopo del encuentro con la amada (Los versos del capitán (1952), Cien sonetos de amor (1959), ya la realización amorosa plena), y segundo, el cronotopo idilico y sociocostumbrista manifestado ahora en la serie de las Odas elementales (1954), donde se destaca lo armónico de los objetos naturales y humanos en su vertiente o faceta poética. Ambas series cronotópicas hablan de la armonía en lo personal y en la poesía, en la naturaleza y en la sociedad. Con Estravagario (1958) se da un salto cualitativo y cambio de paradigma, al emerger el cronotopo del bufón. Es la primera aparición del sujeto juguetón que permite el ingreso de la risa y lo no serio a la discursividad poética.

Entre 1958 y 1964 (y también posteriormente), comienza a primar el cronotopo autobiográfico, de índole memorialística, en Memorial de Isla Negra, de 1964. Este es un cronotopo de lo real del autor y un cronotopo de la rememoración del camino vital realizado. El itinerario de las vidas realizadas es recorrido otra vez. La historia personal es releída y reescrita. Nuevamente vuelve el cronotopo de la novela de formación (pero ya no social como en Canto general, sino que personal y subjetivo, pero en interacción con los otros), de la aventura y de las pruebas (los episodios decisivos), al primer plano. Especial es el caso de La espada encendida (1970), con un cronotopo idílico (mujer y hombre en armonía edénica con la naturaleza, aislados del mundo apocalíptico) y de caballerías, de aventura maravillosa.

Entre 1964 y 1973 se alternan en la obra de Neruda los cronotopos autobiográfico y sociocostumbrista. El uno volcado hacia la persona, el otro, hacia la sociedad. Los libros sobre Cuba o Estados Unidos son de la línea crítica a la sociedad, mientras que, en la obra póstuma, los libros de reflexión personal siguen al cronotopo autobiográfico. Si uno mira el conjunto de la obra, esta apertura hacia lo microsocial (el sujeto personal) y lo macrosocial 
(el sujeto colectivo) está balanceada a lo largo de la sucesión de las diversas etapas y también en el conjunto final de la obra póstuma (incluyendo Confieso que he vivido, 1974).

\section{LAS CUATRO ETAPAS DEL REALISMO NERUDIANO}

La mirada a la obra de Neruda desde la perspectiva del gran tiempo concluye aquí con una última aproximación, basada en "La novela de la educación y su importancia en la historia del realismo", un artículo de Bajtin de 1938, que es, quizás, el único que sobrevive de su magna obra (700 páginas) sobre la Bildungsroman (v. Bajtin, Estética). Aunque se refiere a la novela, Bajtin plantea que el tema principal de su trabajo es el tiempo-espacio y la imagen del hombre, y el criterio central es la asimilación del tiempo histórico real y del hombre histórico. Esta tarea es principalmente de carácter histórico-literario.

Estos han sido los propósitos del presente trabajo. Para concluir, sugerimos que la secuencia novelesca en Occidente, según Bajtin, es la misma que adquieren las obras de Neruda. El primer tipo es el vagabundeo. El protagonista es un punto que se mueve en el espacio. Esto agrupa la obra nerudiana teniendo al poeta como centro desde 1915 hasta la experiencia en Oriente (1932), e incluso la Segunda residencia (1935). Es lo que Bajtin denominó el realismo grotesco de Neruda en su libro sobre Rabelais (el mundo al revés, el carácter distorsionado de la realidad, lo negativo advertido por Amado Alonso).

El segundo tipo es la novela de las pruebas, es decir, los obstáculos que debe sortear y superar el héroe. Esto comienza ya en Santiago, en 1920, y tiene un largo desarrollo, hasta 1952, fecha en que se publica Los versos del capitán. Obviamente, el sentido de las pruebas cambia en 1936, con la "conversión". Antes del 36, el sujeto permanece más o menos invariable, pero la "normalidad" del desencuentro y de las pruebas cambia mediante una serie de acontecimientos extraordinarios (la Guerra Civil Española).

El tercer tipo en la modalidad de construcción gradual de un realismo más complejo, histórico, acontece mediante lo biográfico y lo autobiográfico, que ya está presente en "Explico algunas cosas" y en "Alturas de Macchu Picchu", pero que comienza con Los versos del capitán (anónimamente, 1952) y alcanza su clímax en Memorial de Isla Negra (1964). Esta veta nunca más desaparecerá. El sensorialismo personal del biografismo juvenil es, sin embargo, muy diferente a la rememoriación planificadamente autobiografista de la etapa final nerudiana. Partes del Canto general pueden ser entendidas 
como la historia (auto)biográfica de la saga familiar a la cual pertenece el poeta (autofiliación).

Ya se han tocado los diferentes énfasis en el mundo interno y el mundo externo nerudiano, que son el eje de su obra. El cuarto tipo de modalidad discursiva es el más amplio, equilibrado y complejo: la novela de educación, con su eje en el crecimiento esencial del hombre. Se trata de una unidad dinámica de la imagen del protagonista. El tiempo (y el espacio) ingresa articuladamente al sujeto; persona y realidad se equilibran; la experiencia acaecida se concreta como aprendizaje; el héroe es un sujeto cambiante pero equilibrado frente a las circunstancias.

En la obra póstuma, con la inminencia de la muerte ('el cronotopo innominado', según nuestra denominación), se alcanza la más alta forma del realismo, que no es polifónico aún, sino que dialógico: el desarrollo humano se concibe en una relación indisoluble con el devenir histórico. La transformación del hombre se realiza dentro del tiempo histórico real. De allí la importancia de las Memorias de Neruda, Confieso que he vivido, concluidas casi al inicio de su delirio y agonía.

Realismo dialógico: interacción entre el sujeto y el mundo, algo que toda la crítica nerudiana ha caracterizado como fundamental en su escritura ${ }^{4}$. Diálogo entre la interioridad y la exterioridad, entre el tiempo psíquico y el tiempo histórico, entre la mismidad y la otredad. El hombre, según Bajtin, se localiza entre dos épocas (la vida y la muerte); el héroe se transforma junto con el mundo y se ve obligado a ser un nuevo tipo de hombre.

De allí el sentido final de la experiencia escritural última de Neruda: integración de los sujetos, de los tiempos, de los espacios, de las acciones. Ser y devenir siendo uno solo. Equilibrio, estoicismo, convergencia, aceptación de la verdad última.

\section{BIBLIOGRAFÍA}

Bajtin, Mijail. "De los apuntes 1970-1971”. Estética de la creación verbal. México: Siglo XXI, 1982. 200-247.

Problems of Dostoevsky's Poetics. Trad. C. Emerson. Minneapolis: University of Minnesota Press, 1984.

4 Sobre las etapas de la crítica nerudiana, véase Jofré, Pablo Neruda: Residencia en la tierra. 
"Formas del tiempo y el cronotopo en la novela". Problemas literarios y estéticos.

Trad. A. Caballero. La Habana: Editorial Arte y Literatura, 1986. 269-468.

Jofré, Manuel. Pablo Neruda: Residencia en la tierra. Santiago: ARCIS / Girol Books (Ottawa), 1987.

Pablo Neruda: de los mitos y el ser americano. Santo Domingo (R:D): Ediciones Ferilibro, 2004. 